\title{
Track PVB membrane based on irradiation with high energy protons
}

\author{
Muhammad Shadman, ${ }^{12}$ Farhood Ziaie, ${ }^{2 *}$ Saeid Yeganegi, ${ }^{1}$ Behrooz Niazi, ${ }^{2}$ Farshid \\ Ziaee, ${ }^{3}$ Abbas Majdabadi ${ }^{2}$ \\ ${ }^{1}$ Department of Physical \& Inorganic Chemistry, Mazandaran Uni., Babulsar, Iran. \\ ${ }^{2 *}$ Nuclear Science \& Technology research Institute, Agricultural, Medical and \\ Industrial Research School, P. O. Box 31485-498, Karaj, Iran, tel: +982614424073; \\ fax:+982614464058; email: fziaie@nrcam.org \\ ${ }^{3}$ Iran Polymer and Petrochemical Institute, P. O. Box 14965-115, Tehran, Iran.
}

(Received: 12 August, 2008; published: 21 June, 2009)

\begin{abstract}
One of the methods of polymeric material modification is based on the irradiation with accelerated ions. Chemical etching of ion tracks in polymers is a method which is widely used in the fabrication of micro/nano-structures with predetermined characteristics. For the present study polyvinyl butyral (PVB) porous membrane was prepared by track-etching technique. Commercially available PVB film was irradiated by $30 \mathrm{MeV}$ energy protons to form latent tracks, and then etched chemically by potassium hydroxide $(\mathrm{KOH})$ with the different normalities. The etching rate of PVB was related to the concentration of etching reagent, temperature and time. The porous size and shape were investigated by scanning electron microscope (SEM) after and before etching. Relationships in between the etching rate and the etching parameters were established from experimental data, and can be used to control the pore size of PVB track-etch membrane. The Pore sizes and their structures were evaluated by optical microscope (OM) and scanning electron microscope (SEM). The results have shown the micro/nano-pores formation in the PVB films. The physico-chemical properties of the irradiated samples were investigated and compared with the un-irradiated one using the FTIR and DSC and DTG systems, as well.

Key words: Track Membrane, high-energy protons, nonporous, chemical etching, polyvinyl butyral.
\end{abstract}

\section{Introduction}

The goal of developing polymeric membranes is to form a film with the least amount of impurities with the highest physical properties and chemical resistance. Therefore, the improvement of chemical process for increasing the purity of wide variety of materials have been developed, but the best techniques for this regard are high energy irradiation to pure polymer materials to produce porous media. A polymeric membrane may be generally defined as a membrane film having transport properties comparable with those of the common liquid ionic solutions. High-energy ion irradiation is one of the methods for modification of polymer properties [1] and fabrication of polymeric porous membranes [2]. The production of porous membranes stands out among other applications of the track-etch technique as a micro and nano filter. Temperature, $\mathrm{pH}$ and etching time that experimentally should be defined are effective factors to production of pores [3]. Also varying irradiation parameters such as radiation source, particle energy and irradiation time help to control the pores structures [1]. Recently extensive studies demonstrated the preparation of ion-track 
membranes of some polymers such as polyethylene-terphethalate (PET), polycarbonate (PC), cellulose nitrate, CR-39 and subsequent chemical etching with suitable enchants $[4,5]$. The nuclear track micro/nano filter is a kind of novel material used successfully in various fields such as precise separation of biological cells, filtering of polluted air steams, microelectronics, pharmaceutical, biotechnology, waste water recycling and even fuel cells, etc. [6-10]. Track-etch membranes offer distinct advantages over conventional membranes due to their precisely determined structure. Their pore size, shape and density can be varied in a controllable manner depending on the chemical etching process and irradiation time, so that a membrane with the required transport and retention characteristics can be produced. Ionizing particles passing through certain polymers or electrical insulators displace electrons and produce localized chemical changes along the path that particle travels. When the trajectory of the particle is defined by permanent physical or chemical damages, the disrupted regions surrounding the particle path can be chemically etched at faster rate than the surrounding undamaged material to produce microscopically visible tracks. Registration of tracks occurs if the energy of the particle is higher than a lower threshold value, which is different for various ions and detectors [11]. In the present study, we selected polyvinyl butyral (PVB) because of its strong binding, toughness and flexibility. Therefore, nuclear track micro/nano filters have been developed by exposing PVB films to $30 \mathrm{MeV}$ protons and subsequently followed by chemical etching with alkaline solution. The effects of temperature, $\mathrm{pH}$ and etching time on the pores size were investigated and discussed. The physico-chemical properties of the irradiated samples were investigated and compared with the un-irradiated one using the FTIR and TGA systems.

\section{Results and discussion}

Figures 1 and 2 show the porosity images created by high-energy protons, taken by the OM and the SEM systems. The pores sizes were measured using the different magnifications and the average value for each film sample was calculated.
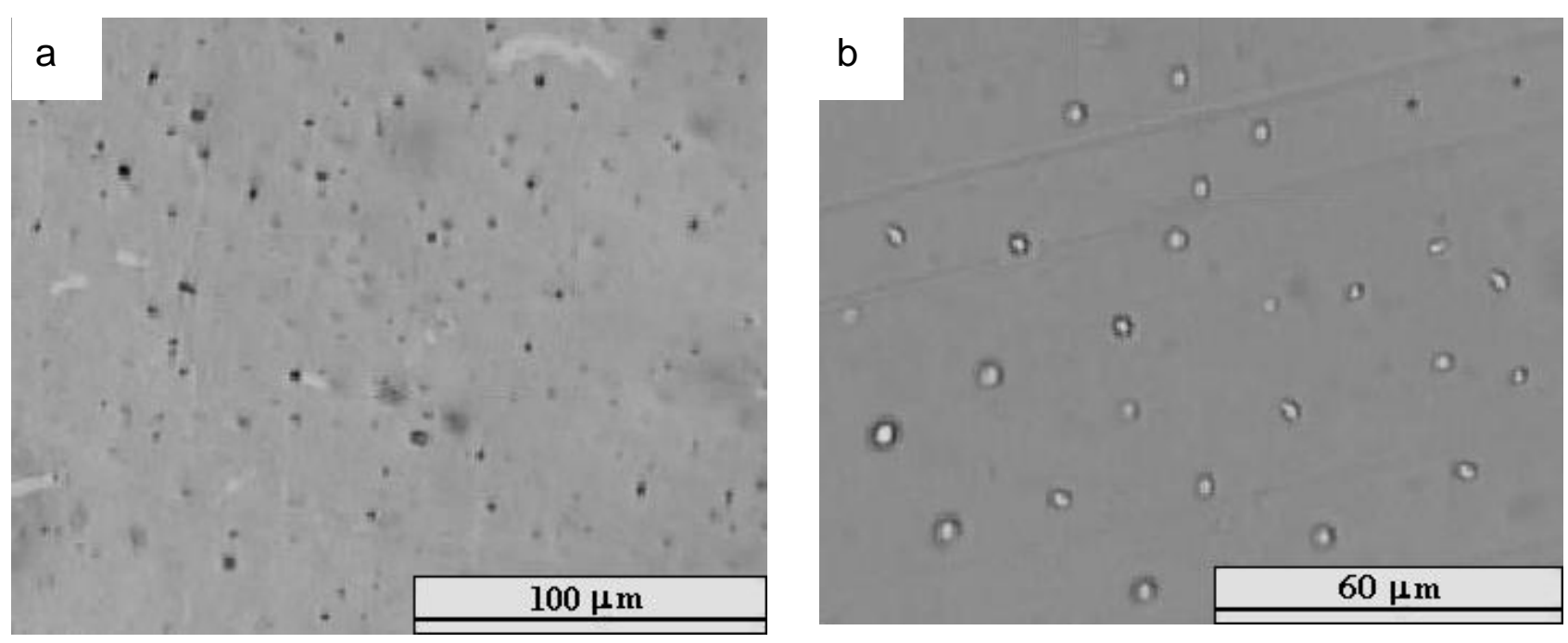

Fig. 1. OM images of the PVB films etched in $6 \mathrm{~N}$ reagent at the etching time of a) 15 $\min$, b) $1 \mathrm{~h}$. 


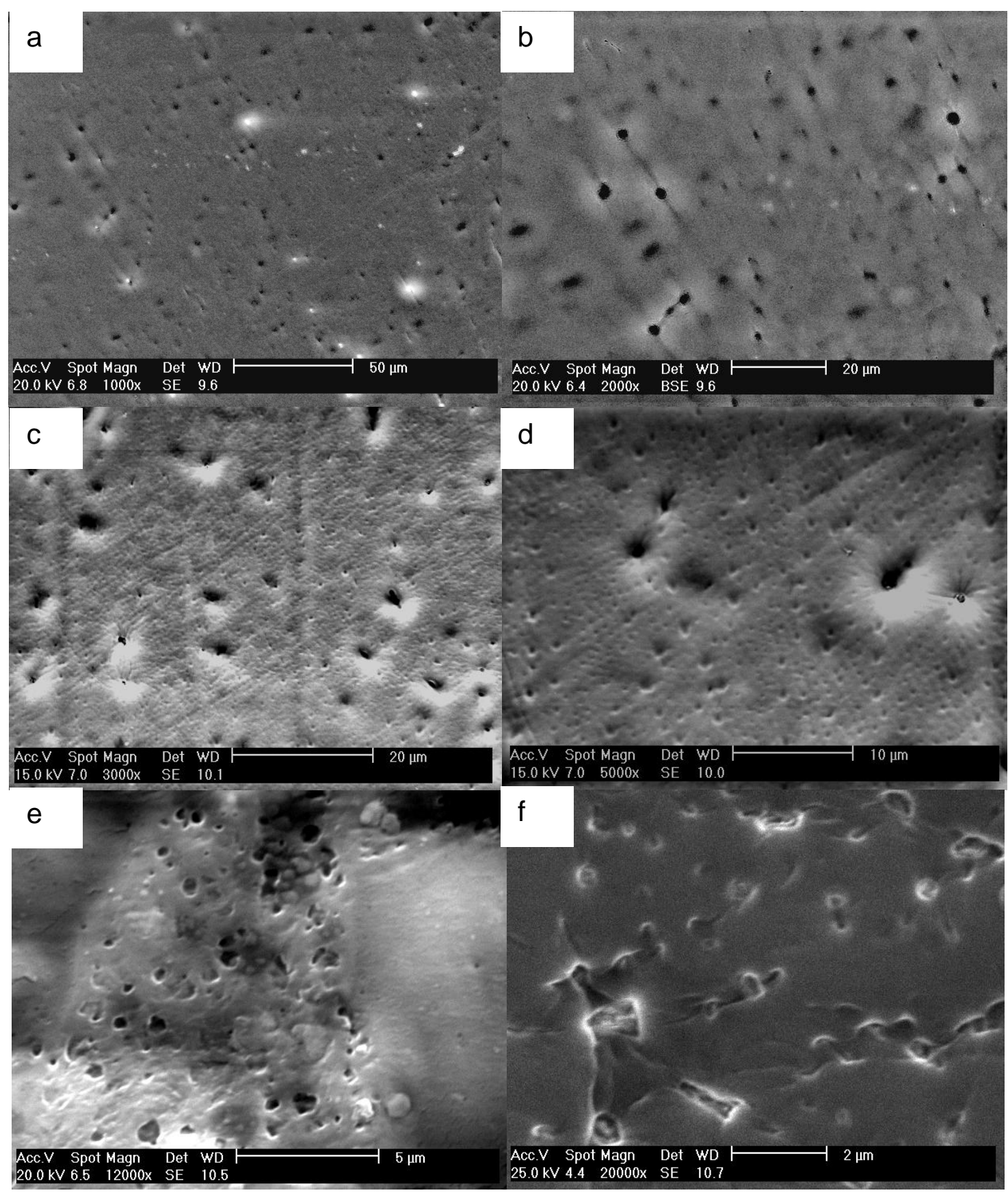

Fig. 2. SEM images of the PVB films etched in $6 \mathrm{~N} \mathrm{KOH}$ at the etching time of 5 min (a); $2.5 \mathrm{~min}$ (b); $1 \mathrm{~min}$ (c, d and d); $30 \mathrm{~s}$ (e and f).

\section{Effect of etching parameters}

Figure 3 shows the variation of pores diameter against the etching time for different normalities of the etching solutions at $60{ }^{\circ} \mathrm{C}$. To see the precise behaviour of the low diameter pores, the scale was changed and demonstrated in Figure 4.

It can be seen clearly that by increasing the etching time up to 1 hour, the pores diameters increases, where, further increase cause decrease in the pore size and finally lead to constant values. The pores sizes at the same etching time are higher for $6 \mathrm{~N}$ solution in comparison to the other one. 


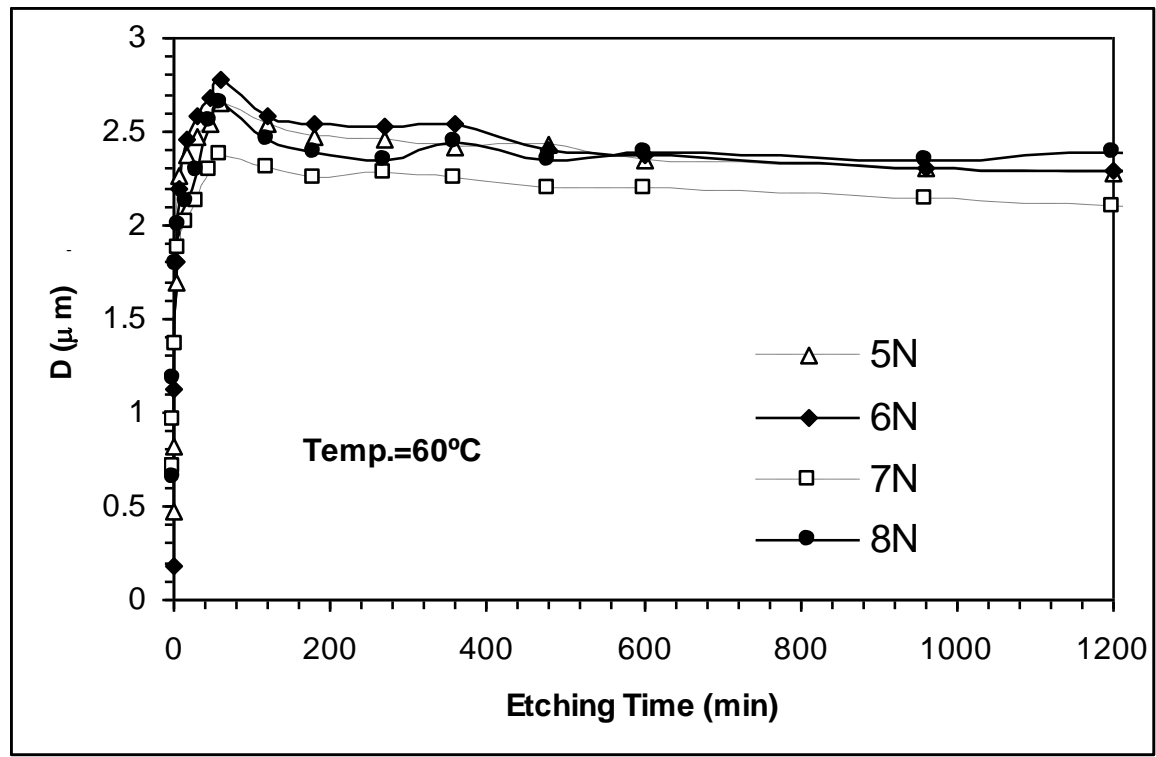

Fig. 3. Variation of pores diameters against the etching time for different normalities of the etching solutions at $60^{\circ} \mathrm{C}$.

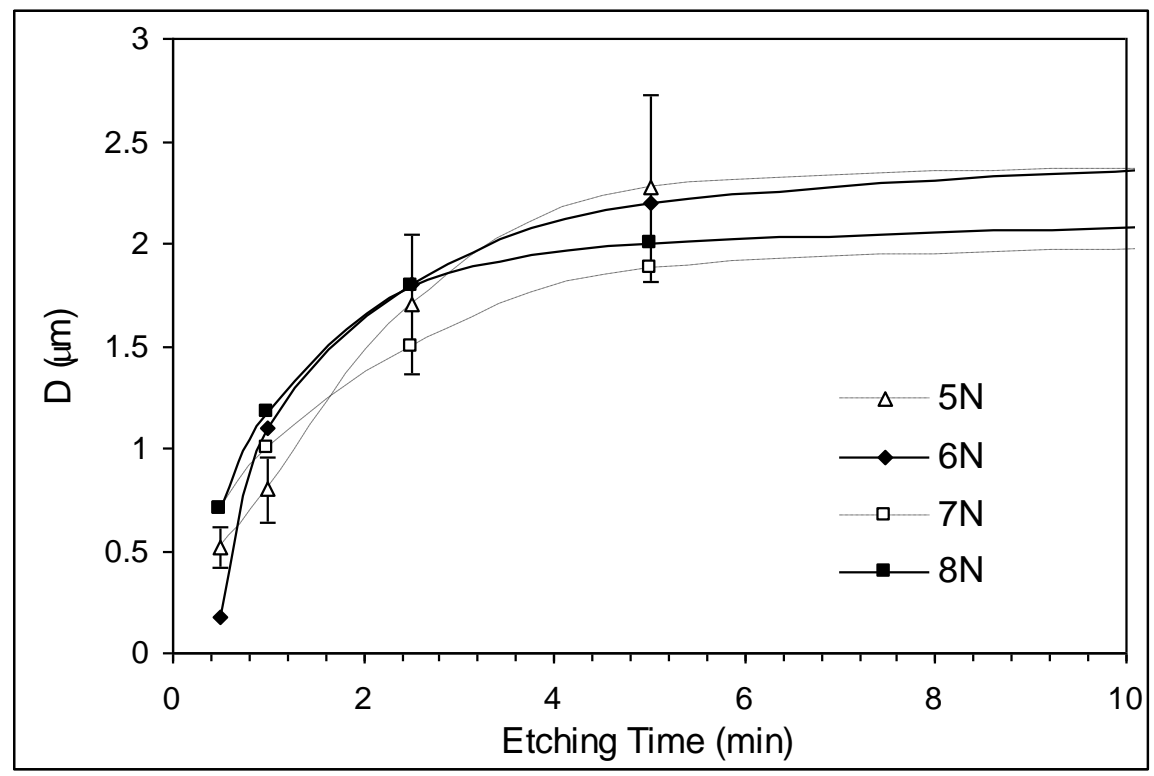

Fig. 4. Fig. 3 with the different scales (the error bars are close to each other for all sets of results).

In fact there are two phenomenon of etching and annealing (due to the etching temperature), which behave in opposite direction. At low etching time, the etching process is dominant while at the higher time the annealing will be dominant process. It is clear that the pore size increases due to the etching, while, annealing process act vice versa [1]. The fact is that the annealing of the damage trails at elevated temperatures presumably occurs via the diffusion of atomic defects through the crystal lattice or the movement of molecular fragments within a polymer. Interstitial atoms can then recombine with lattice vacancies, and broken molecular chain may rejoin and various active species recombine. The detail of such processes is not fully 
understood [1]. Therefore, one could expect to obtain a maximum value during the time, along the curves demonstrated in the recent figures.

On the other hand, the variation of pores diameters against the solution concentration was investigated and the results are demonstrated in Figure 5.

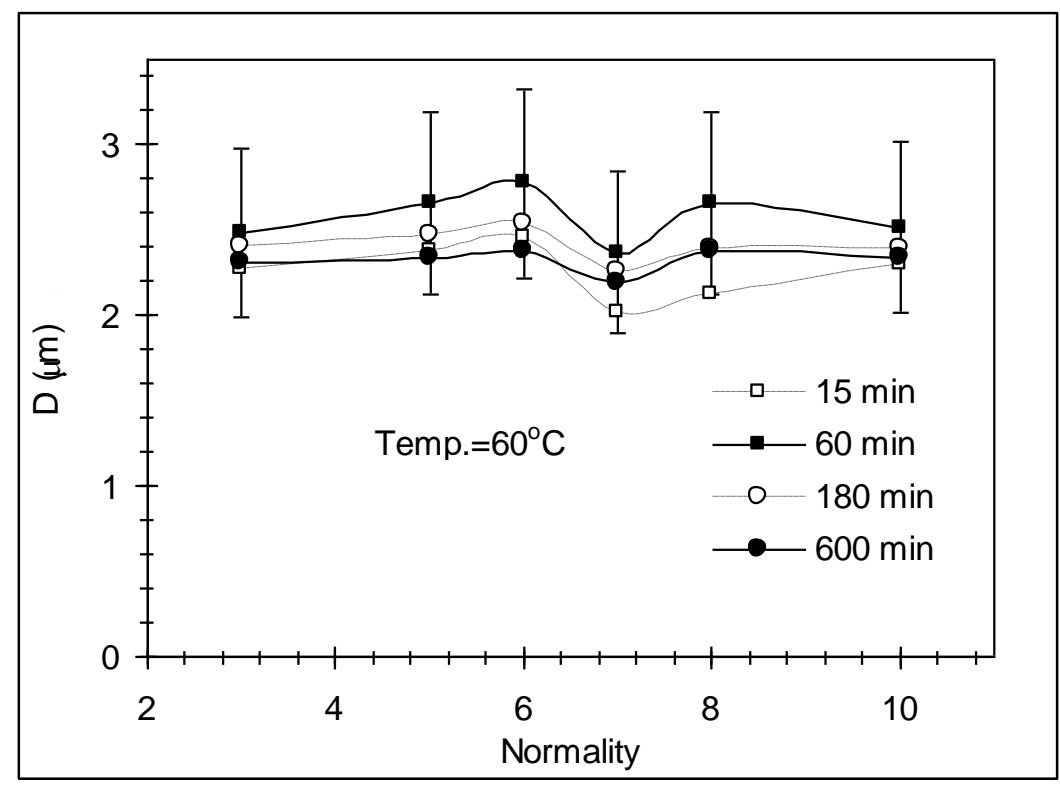

Fig. 5. Variation of pores diameters as a function of etching solution concentrations at $60{ }^{\circ} \mathrm{C}$ of etching temperature (the error bars are close to each other for all sets of results).

In this figure, the experiments were performed in a constant etching time of 15,60 , 180 , and $600 \mathrm{~min}$. The result shows that the maximum pore size could be reached using the $6 \mathrm{~N}$ etching solution concentration, whereas it would be minimum in $7 \mathrm{~N}$ one. The temperature effect was also investigated in this work. Figures 6 and 7 show the variation of the pores size versus etching process temperature for the different etching time of $15,30,45$, and $60 \mathrm{~min}$. It can be seen a bit of increment in $6 \mathrm{~N}$ solution at $60{ }^{\circ} \mathrm{C}$ (Figure 6) and a bit of decrement in $7 \mathrm{~N}$ solution at $60{ }^{\circ} \mathrm{C}$. These results are in agreement with those obtained in Figure 5. However, in the author's view the variations are not very considerable due to the range of uncertainty level, which here is estimated at about $20 \%$. In other words, in the applied temperature range it was not expected to see the pores size variation due to the limitation of the sample thickness in our experiments, where, temperature raising would decrease the pore size due to the mentioned annealing process effect [1]. Thus, this variation could not be very reliable and needs further consideration and precision in the experiment.

Much work has been carried out on the dependence of the etching properties of some plastics on the concentration and temperature of the etchant. Authors have shown a very strong dependence of the parameter $\mathrm{V}\left(=\mathrm{V}_{\mathrm{T}} / \mathrm{V}_{\mathrm{B}}\right.$, where $\mathrm{V}_{\mathrm{T}}$ is track etching rate and $V_{B}$ is bulk etching rate) on the molarity of the etchant and on the temperature of the etching for the given molarity $[12,13]$. These results signify that it is possible to greatly alter the response of a given plastic simply by changing the molarity or temperature of the etchant. The value of $\mathrm{V}$ goes up at first, but, after a 
broad maximum, depending on the particle energy it began to decline again. The decline may be attributed to the slowing down of the removal of etch products from deep track holes at high etchant concentrations. On the other hand, the particle energy increasing causes the decrease of the mentioned maximum of the $\mathrm{V}$ ratio. Therefore, due to the high energy proton used in our work, it was not expected to obtain a considerable maximum along the obtained curve.

It was also found that the ratio $\mathrm{V}$ in Lexan rise with increasing concentration of Lexan etch products in the etching solution. Then the etchant got saturated with these etch products and finally the $\mathrm{V}$ ratio is decreased [14].

These observations also imply that careful work is required in order to establish the optimum etching conditions for each batch of plastic for obtaining the desired results.

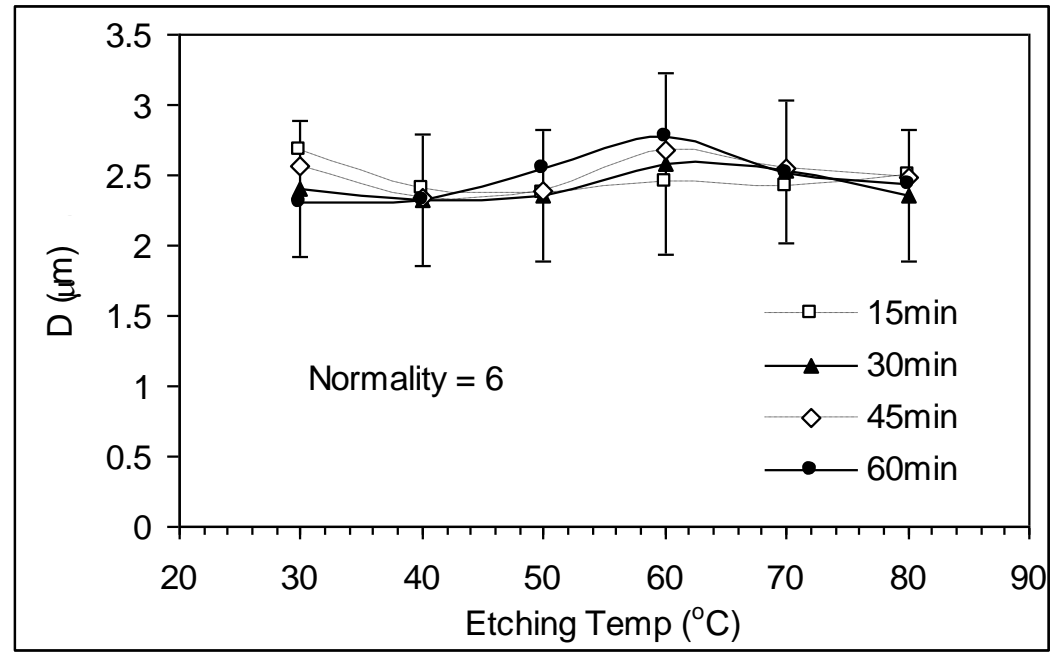

Fig. 6. Variation of pore diameter as a function of etching temperature (the error bars are close to each other for all sets of results).

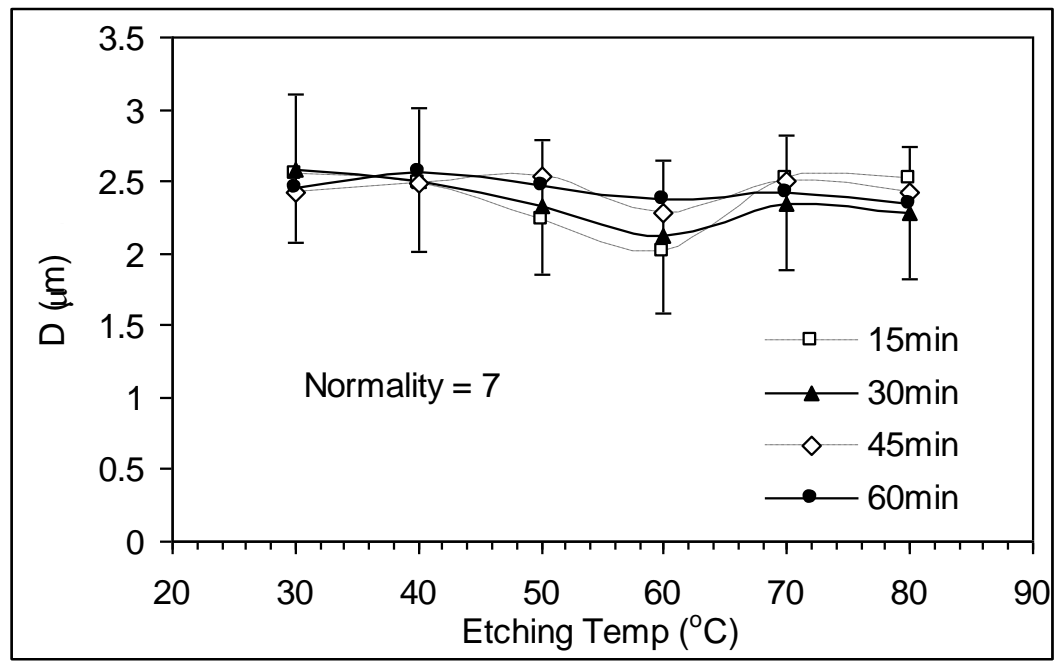

Fig. 7. Variation of pore diameters as a function of etching temperature (the error bars are close to each other for all sets of results). 


\section{Physico-chemical characteristics}

Physical and chemical properties of polymeric membranes are important for accurate flow control, separation and diffusion of multi-component mixtures [15-17]. Admittedly, polymers being used to fabricate membranes for gas separations must have superior mechanical and transport properties. Gas diffusion coefficients typically increase appreciably with increasing temperature when the polymer does not undergo thermally induced morphological rearrangements such as crystallization over the temperature range of interest, since both diffusivity and solubility coefficients are temperature dependent [18]. Thus, stability of mechanical and chemical characteristics of polymeric membranes is interdependent.

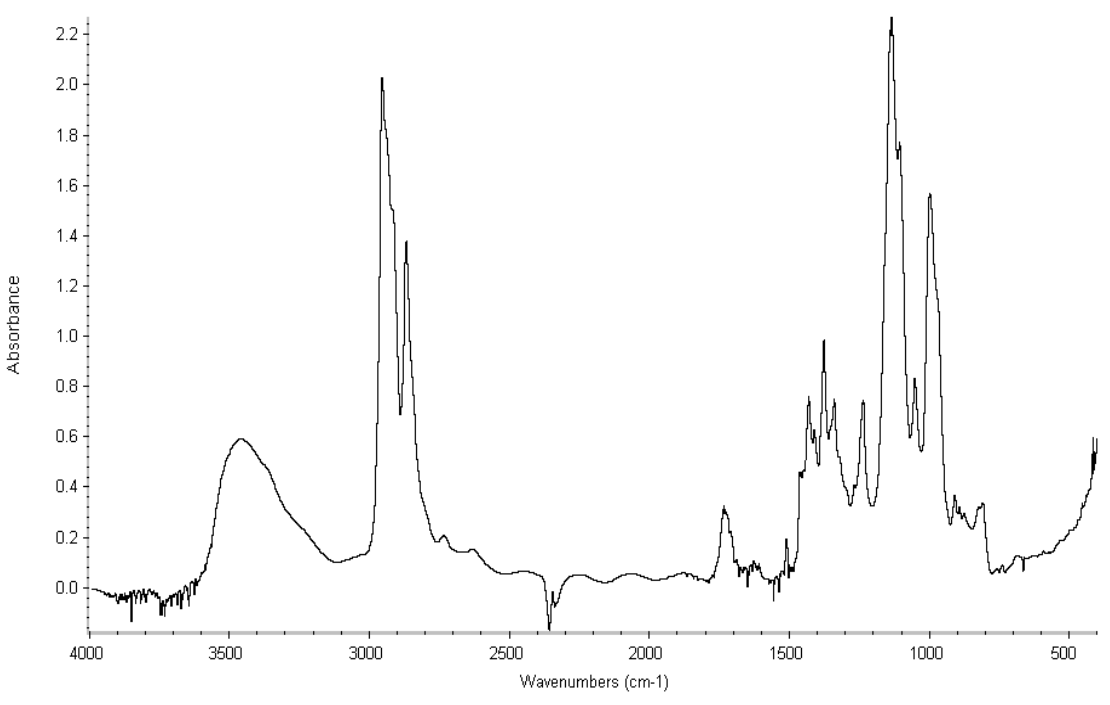

Fig. 8. FTIR spectra of the PVB sample before irradiation.

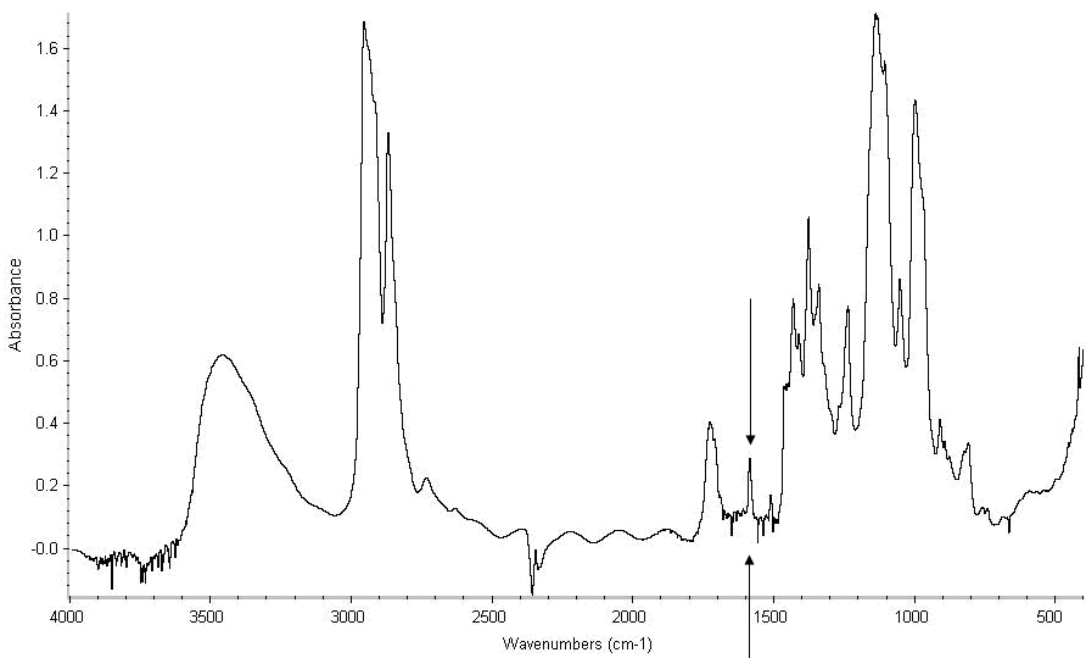

Fig. 9. FTIR spectra of the PVB sample after irradiation under the high $30 \mathrm{MeV}$ protons. The new peaks at $1600 \mathrm{~cm}^{-1}$ help explain the formation of new C-C bond in irradiated film.

During irradiation of PVB film, some bonds of polymeric chain are broken, and may generate free radicals that after chemical etching, cause new bonds that are different from polymeric common bonds. This issue can be followed-up by FTIR technique. 
Figures 8 and 9 illustrate the FTIR spectrums of PVB films before and after irradiation, respectively. The formation of new $\mathrm{C}-\mathrm{C}$ bond in irradiated film corresponded to $1600 \mathrm{~cm}^{-1}$ in Figure 9 shows stability and tenacity of irradiated PVB film in comparison with un-irradiated one and this membrane can be more resistible to high pressure gas or liquid permeability.

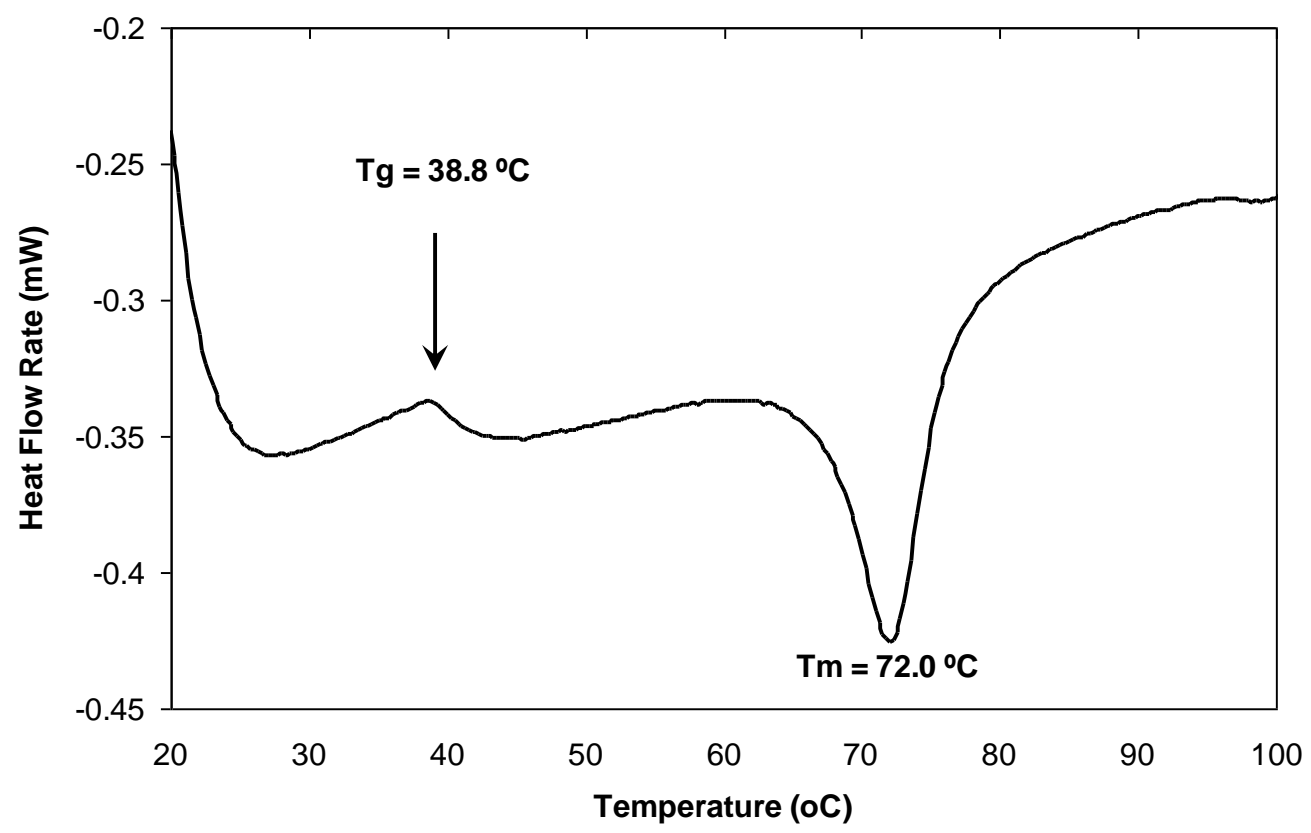

(a)

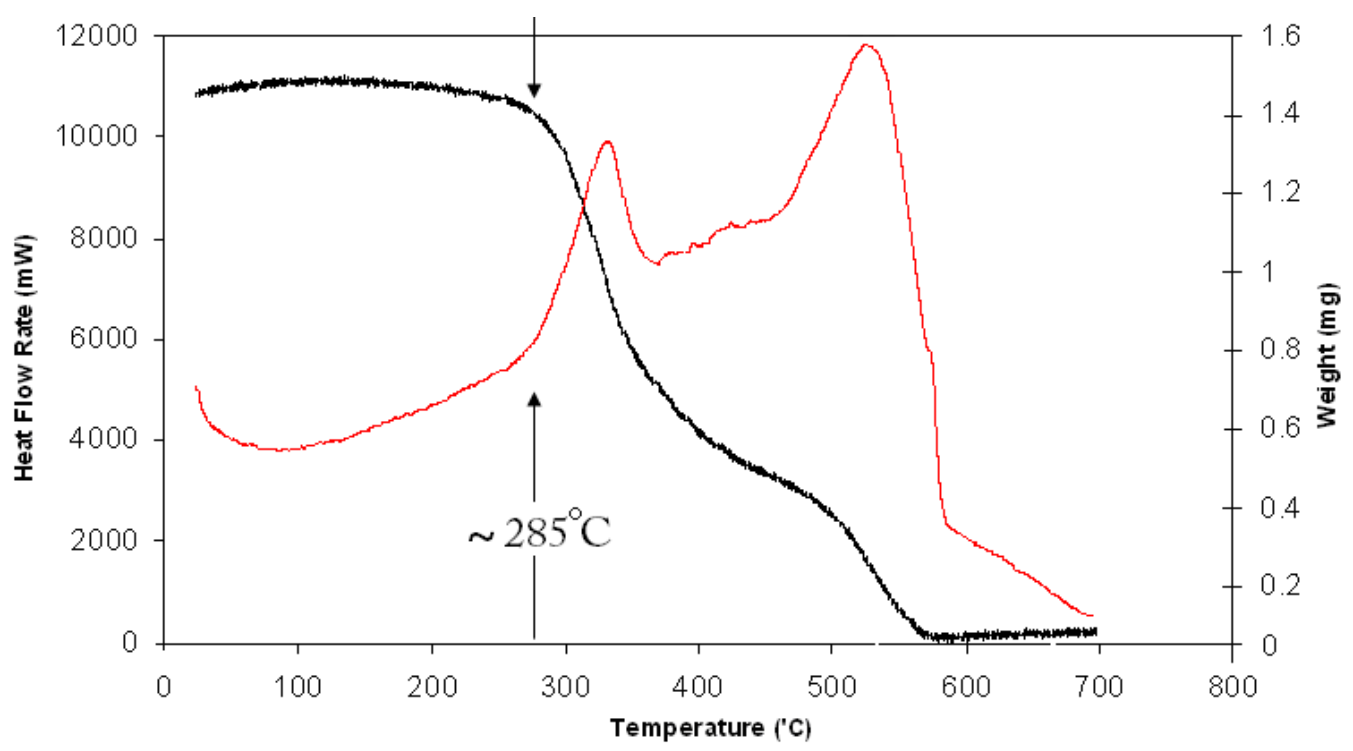

(b)

Fig. 10. DSC (a) and TGA (b) thermo-gram for PVB sample before irradiation.

Furthermore, thermal properties of PVB samples were investigated. Figures 10 and 11 illustrates the DSC and TGA thermo-gram for PVB samples before and after irradiation, respectively. As can be seen in Figures 10, the non-irradiated PVB sample shows glass temperature, $T_{g}$ of $38.8^{\circ} \mathrm{C}$ and degradation temperatures, $T_{d}$, in 
two stages, the first about $285{ }^{\circ} \mathrm{C}$ and the second nearly $500{ }^{\circ} \mathrm{C}$. In this work, the temperature range of main degradation is determined to be $285^{\circ} \mathrm{C}$. The values of $\mathrm{T}_{g}$ and $T_{d}$ of irradiated samples were determined as $55^{\circ} \mathrm{C}$ and $315^{\circ} \mathrm{C}$, respectively. $T_{d}$ increases for protons beam irradiated samples indicating an increase in thermal stability of the polymer. The increasing decomposition temperature, $T_{d}$, is taken into account as a sign of the higher thermal resistance of samples which can be attributed to the dominant cross-linked PVB formation versus the opposite process of chain scission. The resulted values of $T_{g}$ also confirm this interpretation.

The irradiation time in this work was very low, while at higher irradiation time decreasing decomposition temperature could have happened due to the breakage of pervious cross-link bonds.

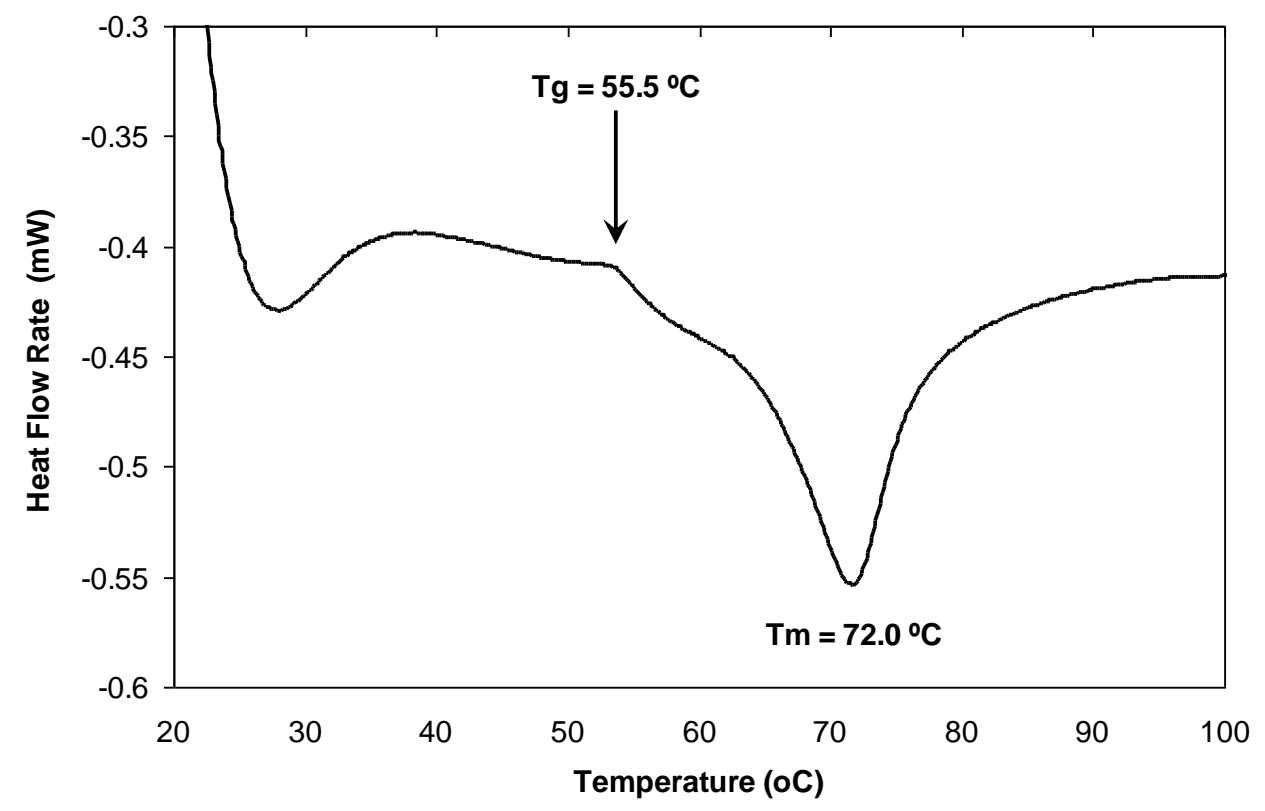

(a)

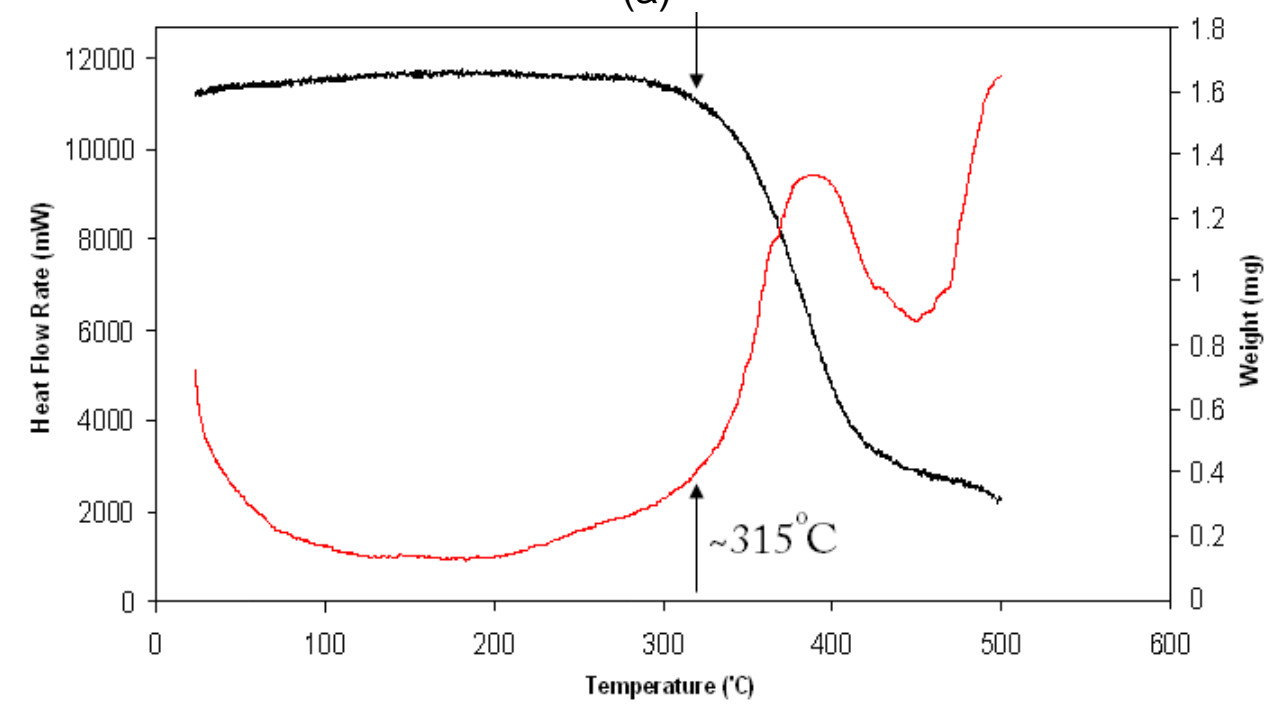

(b)

Fig. 11. DSC (a) and TGA (b) thermogram for PVB sample irradiated under the high $30 \mathrm{MeV}$ protons. 


\section{Experimental part}

\section{Materials}

The polyvinyl butyral (PVB) film with $20 \mu \mathrm{m}$ of thickness made by Ris $\varnothing$ Co., Denmark, were used in this experiment. The $\mathrm{KOH}$ with $99 \%$ of purity provided by MERK Company, Germany, was used as the etching reagent.

\section{Sample preparation \& irradiation}

The PVB film samples were cut to pieces of $40 \mathrm{~mm} \times 60 \mathrm{~mm}$. An Aluminium frame with $45 \mathrm{~mm}$ and $75 \mathrm{~mm}$ internal and external diameter, respectively, and $27 \mathrm{~mm}$ of thickness was designed and constructed as the film holder in the proton beam tube. The film holder can be placed in the radiation field in a manner that the film plane is perpendicular to the proton beam direction. Therefore, the latent tracks (not slant) could be created in the film. The films were irradiated about $5 \mathrm{~s}$ with accelerated protons (energy of $30 \mathrm{MeV}$ ) on the Cyclon-30 cyclotron made by IBA Company, Belgium, in Agricultural, Medical and Industrial Research School (AMIRS).

\section{Etching procedure}

Chemical etching of the tracks is a process of pore formation. During chemical etching, the damaged zone removed and transformed into a hollow channel [19-21]. The irradiated samples were cut to a smaller size, etched in $\mathrm{KOH}$ with the different normalities and etching time at the different etching temperature. The formed alkaline sediment on the film surface during the etching procedure were cleaned in distilled water using an Ultrasonic system of FRITSCH Laborette 17 model, and dried in oven for $10 \mathrm{~min}$.

\section{Microscopic technique}

At the first stage the film samples were examined via an optical microscope (OM) OLYMPUS PMG3, made in Japan. A scanning electron microscope (SEM) XL-30 series, made by Phillips Company, Holland, was used to investigate the lower pores size. The magnification of the system can be obtained from $25 x$ to $400000 x$, using the LaB6 filament. The surfaces of the samples were covered with thin gold film prior to the SEM investigations. The pores sizes were measured by the aforementioned apparatuses, using different magnifications and the average hole size for each film was calculated.

\section{Characterization}

Fourier transmission infrared spectroscopy (FTIR) spectra, was carried out on both irradiated and non-irradiated samples in the wave number range of $400-4000 \mathrm{~cm}^{-1}$ using a Shimadzu 8300.

Thermal analyses of DSC \& TGA were performed to illustrate the thermal stability of the samples using a DSC-NETSCH and Shimadzu DTG-50H analyzers with a heating rate of $10^{\circ} \mathrm{C} / \mathrm{min}$ under $\mathrm{N}_{2}$ atmosphere from room temperature up to $700{ }^{\circ} \mathrm{C}$.

\section{Acknowledgements}

The authors of this paper offer their appreciation to Mr. G.R. Aslani and Mr. S. Lahouti for their very useful co-operation. 


\section{References}

[1] Durrani, S. A.; Bull, R. K. Solid State Nuclear Track Detection, Principle, Methods and Applications, 1986, Pergamon press, Oxford, England.

[2] Neerja, Prasher S.; Singh, S. Radiation Measurements, 2007, 42, 2, 135.

[3] Fischer, B. E.; Spohr, R. Reviews of Modern Physics, 1983 55, 907.

[4] Ferain, E.; Legras, R. Nuclear Instruments and Methods in Physics Research B, 2001, 174, 116.

[5] Suzuki, Y.; Yamaki, T.; Koshikawa, H.; Asano, M.; Voss, K. O.; Neumann, R.; Yoshida, M. Nuclear Instruments and Methods in Physics Research B, 2007, 260, 693.

[6] Varobiev, E. D.; Ovchinnikov, V. V.; Shestakov, V. D. Some peculiarities of use of the polymeric nuclear track membranes in clean rooms. 1989, JINR Report 18, 89.

[7] Dauginet, L.; Duwez, A. S.; Legras, R. Demoustier-Champagne, S. Langmuir, 2001, 17, 3952.

[8] De'jardin, P.; Vasina, E. N.; Berezkin, V. V.; Sobolev, V. D.; Volkov, V. I. Langmuir, 2005, 21, 4680.

[9] Rzepka, S.; Neidhart, B. Fresenius J Anal Chem, 2000, 366, 336.

[10] Gopalani, D.; Kumar, S.; Jodha, A. S.; Rita Singh, R.; Khatri, P. K.; Gopal, R. Journal of Membrane Science, 2000, 178, 1-2, 93.

[11] Ho, J.P.Y.; Yip, C.W.Y.; Koo, V.S.Y.; Nikezic, D.; Yu, K.N. Radiat. Meas. 2002, $35,571$.

[12] Green, P.F.; Ramli, A.G.; Hashemi-Nezjad, S.R.; Al-Najjar, S.A.R. ; Ooi, C.M.; Abujarad, F.; Bull, R.K.; Durrani, S.A. In: Proc. $11^{\text {th }}$ Int. Conf. Solid State Nucl. Track Detectors, Bristol, and Suppl. 3, Nucl. Track. Pergamon, Oxford, 1982, 179.

[13] Fowler, P.H.; Amin, S.; Clapham, V.M.; Henshaw, D.L. In: Proc. $10^{\text {th }}$ Int. Conf. Solid State Nucl. Track Detectors, Lyon, and Suppl. 2, Nucl. Track. Pergamon, Oxford, 1990, 239.

[14] Peterson, D.D. Rev. Sci. Instr. 1970, 41, 1214.

[15] Feron, P.H.M.; Jansen, A.E. Performances and Prospects Separation and Purification Technology, 2002, 27, 231.

[16] Chen, J.; Ma, G. MInt. J. Numer. Meth. Eng. 2006, 68, 1052.

[17] Rivin, D.; Meermeier, G.; Schneider, N.S.; Vishnyakov, A.; Neimark A.V. J. Phys. Chem. B, 2004, 108, 8900.

[18] Kulshrestha, V.; Awasthi, K.; Acharya, N.K.; Singh, M.; Vijay, Y.K. Bull. Mater. Sci., 2005, 28(7), 643.

[19] Mahmood, A.; Bashir, S.; Us-Salam, A.; Ul-Haq, F. Turk J. Phys. 2004, 28, 283.

[20] Fleischer, R.L.; Price, P.B.; Walker, R.M. Nuclear Tracks in Solids: Principles and Applications, University of California Press, Berkeley, 1975.

[21] Fleischer, R.L.; Price, P.B.; Symes, E.M. Science, 1964, 143, 249. 